\title{
Freezing of Aqueous Carboxylic Acid Solutions on Ice
}

\author{
Carolyn J. Moll,* Konrad Meister, Jan Versluis, and Huib J. Bakker \\ Cite This: https://dx.doi.org/10.1021/acs.jpcb.9b10462 \\ Read Online
}

ABSTRACT: We study the properties of acetic acid and propionic acid solutions at the surface of monocrystalline ice with surface-specific vibrational sum-frequency generation (VSFG) and heterodyne-detected vibrational sum-frequency generation spectroscopy (HD-VSFG). When we decrease the temperature toward the eutectic point of the acid solutions, we observe the formation of a freeze concentrated solution (FCS) of the carboxylic acids that is brought about by a freeze-induced phase separation (FIPS). The freeze concentrated solution freezes on top of the ice surface as we cool the system below the eutectic point. We find that for freeze concentrated acetic acid solutions the freezing causes a strong decrease of the VSFG signal, while for propionic acid an

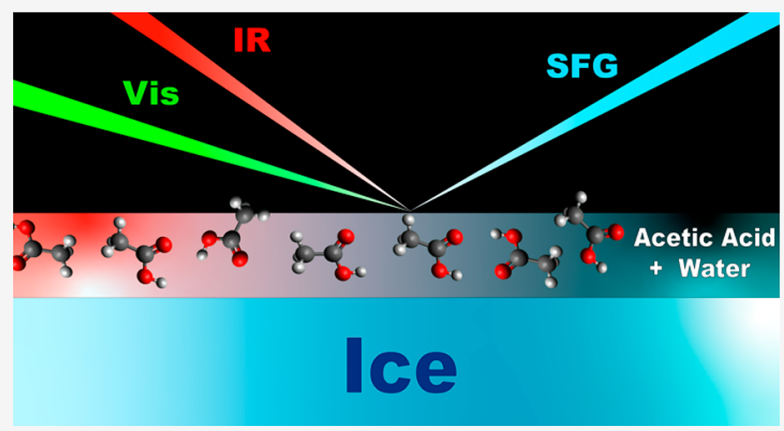
increase and a blue-shift are observed. This different behavior points at a distinct difference in molecular-scale behavior when cooling below the eutectic point. We find that cooling of the propionic acid solution below the eutectic point leads to the formation of hydrogen-bonded dimers with an opposite alignment of the carboxylic acid $\mathrm{O}-\mathrm{H}$ groups.

\section{INTRODUCTION}

Carboxylic acids such as acetic acid (AAc) or propionic acid (PAc) are weak acids that consist of a hydrophilic carboxyl group and a hydrophobic alkyl chain. They are the most abundant oxygenated compounds in the atmosphere and form a major contributor to free acidity in precipitation. ${ }^{1,2}$ The interaction of atmospheric inorganic and organic species, including carboxylic acids with the surface of ice particles, plays an important role in the heterogeneous chemistry of clouds and is thus important in the study of climate change and other environmental issues. It is well-known that during freezing of atmospheric droplets, freeze-induced phase separation (FIPS) and the concomitant freeze concentration of the solutions (FCS) around ice particles occur. These phenomena have a great impact on the surface reactivity, on the physical and chemical properties of ice clouds, and therefore on the climate. $^{3-6}$

Chemical processes occurring at ice surfaces are of great importance to atmospheric chemistry and have attracted significant attention in the past decade. ${ }^{2,7-9}$ Ice surfaces are known to act as sinks for pollutants, a process that considerably changes the properties of the ice surface. ${ }^{10}$ Ice samples with impurities often contain a liquid solution phase that coexists with solid ice over a wide temperature range. ${ }^{5,11}$ It is important that at the interface of the ice surface and the liquid solution, chemical reactions and processes often show characteristics different from those seen in bulk liquid solutions. ${ }^{12,13}$

Here, we use conventional (VSFG) and heterodynedetected vibrational sum-frequency generation spectroscopy (HD-VSFG) to investigate how atmospheric organic species such as AAc and PAc modify the structure and physical and chemical properties of the ice surface.

VSFG is a highly surface-specific technique that is ideally suited for the study of molecules adsorbed at interfaces. ${ }^{14}$ In VSFG, an infrared light pulse and a visible pulse are combined to generate light at their sum-frequency. The generation is enhanced in case the infrared light is resonant with a molecular vibration. VSFG signals can only be generated if the symmetry is broken, for example, at an interface, and are forbidden in the bulk of a centrosymmetric medium. VSFG techniques have successfully been applied to investigate ice surfaces and the freezing of solutions next to solid surfaces. ${ }^{13,15-19}$ By interfering the VSFG signal from the sample with a reference sum-frequency field, we determine the phase of the generated sum-frequency light and thereby the phase of the second-order optical susceptibility $\chi^{(2)}$. The real ( $\left.\mathrm{Re}\right)$ and imaginary (Im) parts of $\chi^{(2)}$ provide direct information on the orientation of the vibrational transition dipole moments at the surface. Thereby HD-VSFG provides unique information on the absolute orientations of the molecules at the surface. ${ }^{20}$

Received: November 7, 2019

Revised: $\quad$ May 12, 2020

Published: May 15, 2020 


\section{MATERIALS AND METHODS}

HD-VSFG Experiments. The setup used for the VSFG experiments is based on a commercial Ti:sapphire laser source, which consists of an oscillator (Coherent "Mantis") and an amplifier (Coherent "UPS"). The oscillator delivers 35 fs pulses centered at $800 \mathrm{~nm}$ that are amplified by using chirped pulse amplification. The resulting pulses have an energy of 3.5 $\mathrm{mJ}$ and a repetition rate of $1 \mathrm{kHz}$. Approximately two-thirds of the laser output is used to pump a home-built optical parametric amplifier (OPA) and a difference-frequency mixing (DFG) stage. The resulting mid-infrared pulses are tunable from 2.5 to $10 \mu \mathrm{m}$ with a bandwidth of $400 \mathrm{~cm}^{-1}$. These pulses have an energy of $10-20 \mu \mathrm{J}$. The other one-third of the 800 $\mathrm{nm}$ laser output is sent through an etalon to narrow the bandwidth to $\sim 15 \mathrm{~cm}^{-1}$. The resulting narrow-band $800 \mathrm{~nm}$ visible light pulses (VIS) and the mid-infrared pulses (IR) are combined at the sample surface in spatial and temporal overlap. The VIS and IR beams are sent onto the sample surface at angles of incidence of $\sim 50^{\circ}$ and $\sim 55^{\circ}$ and focused with lenses with focal lengths of 200 and $100 \mathrm{~mm}$, respectively. The generated and reflected SFG light is directed into a spectrometer and detected by a thermo-electrically cooled electron multiplied charged-coupled device (EMCCD, Andor Technoligies). We also perform a reference measurement where we replace the sample by z-cut quartz. The intensity VSFG spectrum $\left|\chi^{(2)}(\omega)\right|^{2}$ is then obtained by dividing the spectrum measured with the sample by the spectrum measured with the reference z-cut quartz crystal. With this division, we correct the VSFG spectrum for the frequency dependence of the intensity of the input infrared beam. Furthermore, we perform a background subtraction.

VSFG provides information about the spectral dependence of the absolute square of the second-order susceptibility, that is, $\left|\chi^{(2)}(\omega)\right|^{2}$. To obtain the imaginary and real parts of $\chi^{(2)}(\omega)$, the SFG signal of the sample is combined with that of a local oscillator (LO) at the same frequency. The LO is created by focusing the VIS and IR beams on a gold metal surface that leads to the generation of a strong nonresonant SFG signal. The resulting broadband SFG and the reflected VIS and IR beams are then all three directed to the sample surface. Before reaching the sample surface, the LO signal is time delayed by $\sim 1.6$ ps with respect to the IR and VIS beam by passing this beam through a silica plate with a thickness of $\sim 1 \mathrm{~mm}$. All of the beams are refocused by a spherical mirror to the sample surface, where the VIS and IR beam will generate the VSFG signal. Subsequently, the delayed LO-SFG and sample VSFG light are recollimated, sent into the spectrometer, and detected with the EMCCD (Figure 1). The interference of the LO-SFG and VSFG light leads to a spectral modulation. The typical acquisition time of a HD-VSFG spectrum is $120 \mathrm{~s}$.

The measured spectral response is again corrected for the spectral dependence of the input IR beam by dividing the HDVSFG spectrum of the sample by the HD-SFG spectrum of a reference z-cut quartz crystal. In this normalization, it is important to make sure that the surface of the z-cut quartz crystal is at the same height as the sample, as a difference in height will lead to an error in the determination of the phase of the VSFG light and thus of $\chi^{(2)}(\omega)$. By monitoring the position of the VSFG-signal on the EMCCD, we can control the height of the reference z-cut quartz crystal to such an extent that the overall phase uncertainty is $\sim \pi / 10$.

\section{Heterodyne detected SFG}

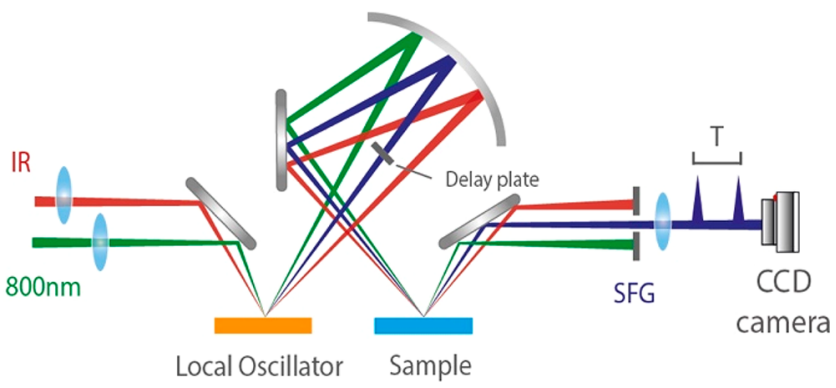

Figure 1. Schematic layout of the used heterodyne detected VSFG. The IR and the $800 \mathrm{~nm}$ beam are focused onto the local oscillator to generate an intense SFG response (LO-SFG) that is delayed by a delay plate. The three beams are refocused on the sample surface where an SFG signal of the sample is produced, which interferes with the LO-SFG signal on the EMCCD camera.

Single Ice Growth, Orientation, and Sample Preparation. For the fabrication of monocrystalline ice, we used the method of seed extraction from the melt. ${ }^{21}$ The ice growing process is started by freezing a monocrystalline ice seed on a precooled cooper pin. The copper pin is cooled to a temperature of $272 \mathrm{~K}$ by using a processor cooler (LDPCV2). Next, the ice seed is dipped into a pan filled with cooled ultrapure water. After $\sim 10 \mathrm{~min}$, the temperature of the copper pin is gradually decreased to a value of $233 \mathrm{~K}$. Sixty minutes later, the ice seed is pulled out from the melt with a velocity of $5 \mathrm{~mm} / \mathrm{h}$. The growing process is stopped after $\sim 10 \mathrm{~h}$, when the ice crystal has reached a height of $\sim 70 \mathrm{~mm}$.

All of the experiments were performed with an ice crystal sample for which the surface is parallel to the basal plane (perpendicular to the main optical axis). To achieve this orientation, the ice is first cut in manageable pieces by using a band saw (Proxxon MBS 240/E) with a nickel-coated blade. The orientation of the crystal pieces is determined using a Rigsby stage following the routine described by Fairbairn. ${ }^{22}$ Subsequently, the surface is scraped with a heated blade to polish the surface, while keeping it parallel to the basal plane. Next, a $\sim 4 \mathrm{~mm}$ thick sample slice is cut off with the band saw perpendicular to the optical axes.

The HD-VSFG measurements are carried out within a liquid nitrogen temperature cooled sample cell that is closed with a $\mathrm{CaF}_{2}$ window. The temperature is controlled by a temperature sensor glued directly to the surface and regulated by a heating foil covered with a copper plate. To avoid heat accumulation and damage to the ice sample, two motors are moving the sample cell constantly in a plane parallel to the surface. To ensure that the ice crystal sample surface was not contaminated, we measured a VSFG spectrum before putting the carboxylic acid solution on the ice crystal.

The carboxylic acid solutions ( $40 \mathrm{wt} \%$ ) are put on the ice surface by using a combination of a clean tissue and a plastic foil clamped in a tweezer. The tissue acts like a sponge to soak up the solution, and the plastic foil smooths the surface after the carboxylic acid is applied. With this technique, the AAc and PAc solutions are placed on the ice surface at temperatures of 265 and $268 \mathrm{~K}$, respectively. The applied layers have a thickness of several tens of nanometers, which implies that they are macroscopically thick on the molecular scale (containing many solution layers) but are sufficiently thin to avoid significant absorption and phase-change effects. To determine 

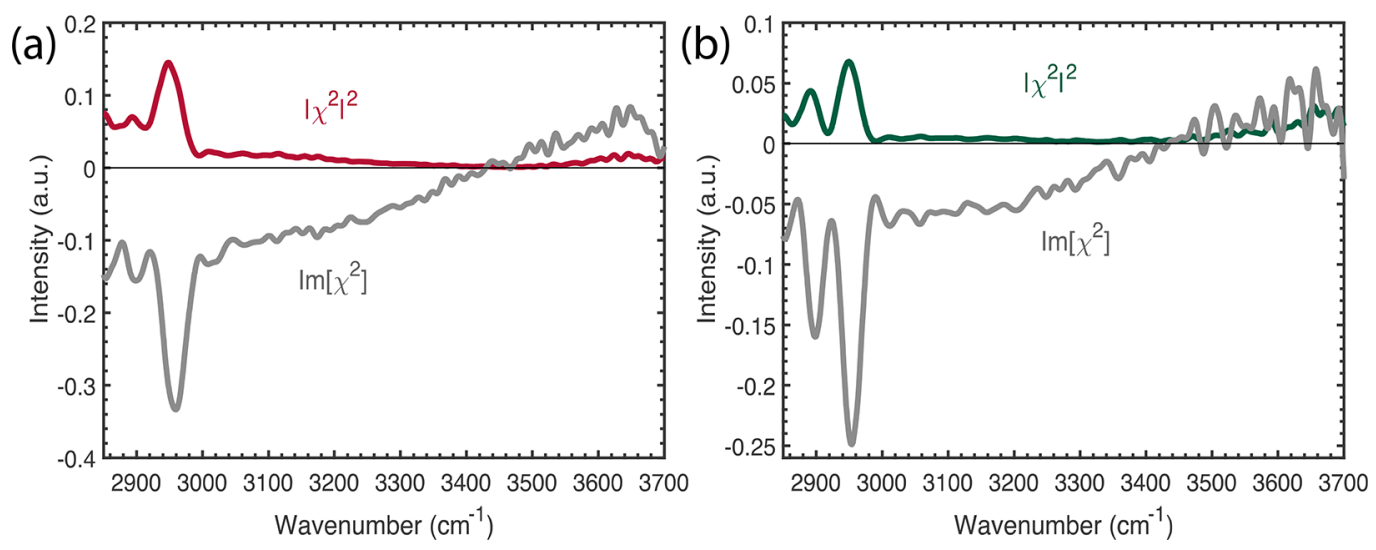

Figure 2. VSFG intensity spectra of aqueous acetic acid (40 wt \%, red) and propionic acid (40 wt \%, green) at the solution-air interface. The corresponding $\operatorname{Im}\left[\chi^{(2)}\right]$ spectrum is shown in gray. All measurements were performed at room temperature (293 K) and in an ssp polarization configuration (s-SFG, s-VIS, p-IR).

the influence of interference effects caused by the solution layer on top of the ice surface, we modeled the VSFG spectrum including these effects (Figure S1a-c). From this modeling, we estimate the thickness of the AAc solution on ice to be $\sim 50 \mathrm{~nm}$ at $268 \mathrm{~K}$ and $\sim 12 \mathrm{~nm}$ at $248 \mathrm{~K}$. Similar values are obtained for the PAc solution on ice. In addition, we used a cooling rate of $\sim 1 \mathrm{~K} / \mathrm{min}$. Each measurement was taken $15 \mathrm{~min}$ after the temperature of interest was reached to allow the sample to equilibrate.

\section{RESULTS AND DISCUSSION}

In Figure 2 we present the $\left|\chi^{(2)}\right|^{2}$ and the $\operatorname{Im}\left[\chi^{(2)}\right]$ spectra of (a) aqueous 40 wt \% AAc and (b) PAc solutions, measured with an ssp polarization configuration (s-SFG, s-VIS, p-IR). The presented $\left|\chi^{(2)}\right|^{2}$ spectra for AAc and PAc are in excellent agreement with the results obtained in previous studies. ${ }^{23-25}$

The $\left|\chi^{(2)}\right|^{2}$ spectrum of the AAc solution spectrum shows a sharp peak at $\sim 2950 \mathrm{~cm}^{-1}$, which we assign to the symmetric methyl stretch vibration. ${ }^{24}$ The $\left|\chi^{(2)}\right|^{2}$ spectrum further shows a very broad signal with maxima at $\sim 3080$ and $\sim 3620 \mathrm{~cm}^{-1}$ that have been assigned to the $\mathrm{OH}$ stretch of the strong hydrogenbonded $\mathrm{OH}$ groups of the carboxylic acids with the water molecules and to the $\mathrm{O}-\mathrm{H}$ stretch vibrations of weakly hydrogen-bonded water molecules, respectively. ${ }^{24}$ However, the $\operatorname{Im}\left[\chi^{(2)}\right]$ spectrum reveals that in fact there exists only a single very broad distribution of $\mathrm{OH}$ stretch frequencies that appears as two separate responses in the $\left|\chi^{(2)}\right|^{2}$ spectrum because the sign of the signal changes at $\sim 3450 \mathrm{~cm}^{-1}$. The change of the sign within the broad band of $\mathrm{OH}$ stretch vibrations reveals that the orientation of the transition dipole moment, and therefore the orientation of the molecules, changes as a function of frequency. The PAc solution spectrum looks very similar to the AAc spectrum and only contains an additional signal at $2880 \mathrm{~cm}^{-1}$ that we assign to the $\mathrm{C}-\mathrm{H}$ stretch vibration of the methylene group.

In Figure $3 a$ and $b$ we show the $\left|\chi^{(2)}\right|^{2}$ and the $\operatorname{Im}\left[\chi^{(2)}\right]$ VSFG spectra of a $40 \mathrm{wt} \%$ AAc solution at the ice surface at temperatures ranging from 265 to $235 \mathrm{~K}$. The $\operatorname{Re}\left[\chi^{(2)}\right]$ spectra of these measurements can be found in Figure S3. We find that at $265 \mathrm{~K}$ the $\left|\chi^{(2)}\right|^{2}$ spectrum looks quite similar to that of an AAc solution-air interface at room temperature. However, an important difference is that the VSFG spectrum of the AAc solution on ice contains an additional weak broad band around $\sim 3160 \mathrm{~cm}^{-1}$. Decreasing the temperature to $255 \mathrm{~K}$ leads to the rise of the additional strong signal centered at $\sim 3160 \mathrm{~cm}^{-1}$ that corresponds to both the response of $\mathrm{O}-\mathrm{H}$ stretch vibration of AAc molecules and the ice surface underneath the AAc solution. Decreasing the temperature also induces an increase of the band at $2950 \mathrm{~cm}^{-1}$ in the intensity VSFG spectra of Figure 3a. Comparison with the $\operatorname{Im}\left[\chi^{(2}\right]$ spectra at different temperatures of Figure $3 b$ shows that this increase in $\left|\chi^{(2)}\right|^{2}$ largely results from the constructive interference of the response of the $\mathrm{C}-\mathrm{H}$ methyl stretch vibration at $2950 \mathrm{~cm}^{-1}$ and the low-frequency wing of the broad $\mathrm{O}-\mathrm{H}$ signal that becomes stronger when the temperature is lowered.

Figure $3 c$ presents the phase diagram for an aqueous solution of AAc. According to this phase diagram, we expect that placing a $40 \mathrm{wt} \% \mathrm{AAc}$ solution at $265 \mathrm{~K}$ on the ice crystal will melt the top layers of the ice surface until a concentration of $\sim 22 \mathrm{wt} \%$ is reached. Lowering the temperature leads to freezing out of water from the AAc solution and thus to an increase of the concentration AAc. Upon lowering the temperature below the eutectic point of the solution (246 $\mathrm{K})$, diffuse light scattering from the ice surface can be observed, which induces a significant decrease of the $\left|\chi^{(2)}\right|^{2}$ signal intensities at 2950 and $3160 \mathrm{~cm}^{-1}$. This strong decrease of the $\mathrm{OH}$-stretch signals upon cooling below the eutectic point is reversible by increasing the temperature again above the eutectic point, as was demonstrated in Figure S4.

In Figure $4 a$ and $b$ we show the $\left|\chi^{(2)}\right|^{2}$ and the $\operatorname{Im}\left[\chi^{(2)}\right]$ VSFG spectra of a $40 \mathrm{wt} \%$ PAc solution placed on the surface of ice at temperatures between 268 and $235 \mathrm{~K}$. The $\operatorname{Re}\left[\chi^{(2)}\right]$ spectra of these measurements can be found in Figure S5. Figure $4 \mathrm{c}$ shows the phase diagram of water-PAc mixtures. At $268 \mathrm{~K}$, the VSFG spectrum looks similar to the VSFG spectrum of an aqueous PAc solution at room temperature. As in the case of AAc, we observe the rise of a strong signal at $3160 \mathrm{~cm}^{-1}$ when we lower the temperature to $255 \mathrm{~K}$. This rise is accompanied by an increase of the $\mathrm{C}-\mathrm{H}$ signals at 2880 and $2950 \mathrm{~cm}^{-1}$ in the intensity VSFG spectra of Figure 4a. Similar to the case of AAc on ice, it is clear from the corresponding $\operatorname{Im}\left[\chi^{(2)}\right]$ spectra (Figure $4 \mathrm{~b}$ ) that this increase results from enhanced constructive interference with the low-frequency wing of the increasing signal at $\sim 3160 \mathrm{~cm}^{-1}$. Lowering the temperature below the eutectic point of the PAc solution $(\sim 245 \mathrm{~K})$ results in a further increase and blue-shift of the signal at $3160 \mathrm{~cm}^{-1}$. The blue-shift of the VSFG signal at 3160 $\mathrm{cm}^{-1}$ upon cooling below the eutectic point of the mixture is 
(a)

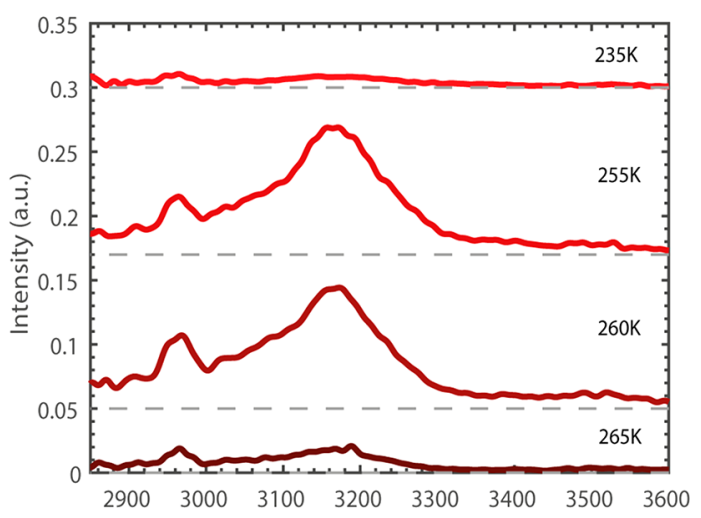

(b)

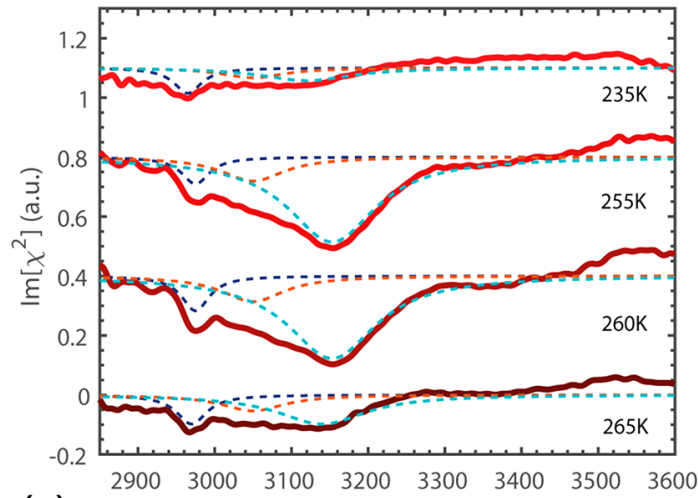

(c)

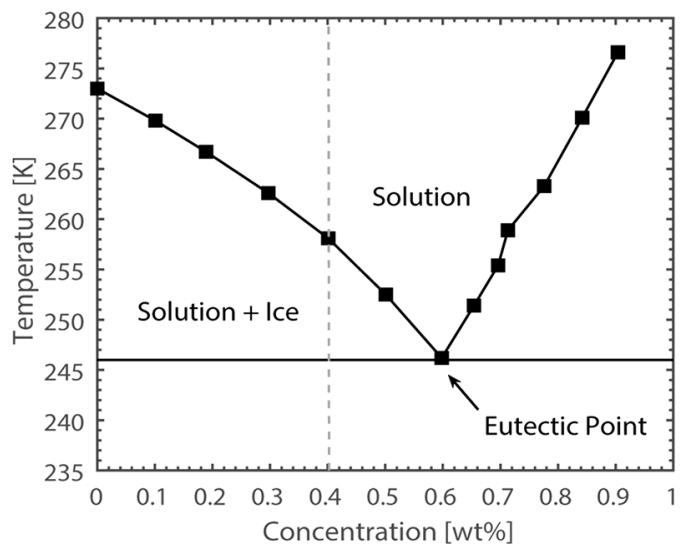

Figure 3. VSFG spectra of aqueous acetic acid (40 wt \%, red) at the surface of ice at different temperatures. (a) The intensity VSFG spectra $\left(\left|\chi^{(2)}\right|^{2}\right)$ and (b) the imaginary part of $\chi^{(2)}$. The colored dashed curves in (b) present the spectral decomposition of the $\operatorname{Im}\left[\chi^{(2)}\right]$ spectra in Lorentzian components. (c) The phase diagram for aqueous solutions of AAc. The data points in (c) were obtained by Barr et al. ${ }^{36}$

reversible upon heating and cooling as shown in Figure S6. Lowering the temperature below the eutectic point also leads to a decrease of the $\mathrm{C}-\mathrm{H}$ signals at 2880 and $2950 \mathrm{~cm}^{-1}$ and a change in the ratio of the two signals in the intensity VSFG spectrum of Figure 4a. This change in ratio is not observed in the $\operatorname{Im}\left[\chi^{(2)}\right]$ spectrum of Figure $4 b$, which implies that it merely reflects a change of the interference effect with the red wing of the broad $\mathrm{O}-\mathrm{H}$ signal.

We performed a spectral decomposition of the $\operatorname{Im}\left[\chi^{(2)}\right]$ spectra of Figures $3 \mathrm{~b}$ and $4 \mathrm{~b}$ in Lorentzian-shaped spectral (a)
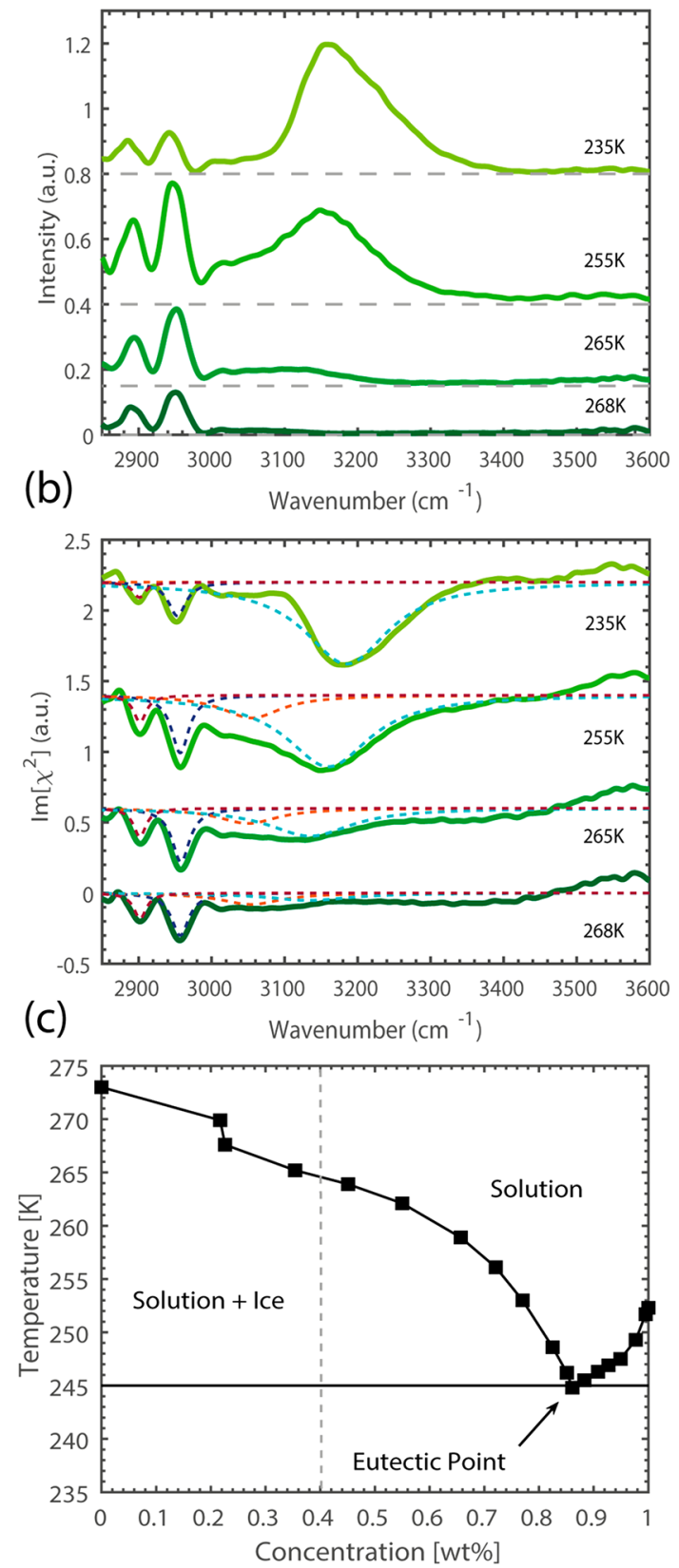

Figure 4. VSFG spectra of aqueous propionic acid (40 wt \%, green) at the surface of ice at different temperatures. (a) The intensity VSFG spectra $\left(\left|\chi^{(2)}\right|^{2}\right)$ and (b) the imaginary part of $\chi^{(2)}$. The colored dashed curves in (b) present the spectral decomposition of the $\operatorname{Im}\left[\chi^{(2)}\right]$ spectra in Lorentzian components. (c) The phase diagram for aqueous solutions of PAc. The data points in (c) were obtained by Barr et al. ${ }^{36}$

components curves. We use three components for the solution of AAc on ice that we assign to the $\mathrm{C}-\mathrm{H}$ vibration of the $\mathrm{CH}_{3}$ group (band at $2950 \mathrm{~cm}^{-1}$ ), the $\mathrm{O}-\mathrm{H}$ vibration of strongly hydrogen-bonded carboxylic acid $\mathrm{OH}$ groups $\left(3080 \mathrm{~cm}^{-1}\right)$, and the $\mathrm{O}-\mathrm{H}$ vibration of ice underneath the AAc solution (band at $3160 \mathrm{~cm}^{-1}$ ). This signal has a frequency position and spectral shape similar to those of the main component of the VSFG spectrum of the bare ice-air interface (Figure S7) and has been assigned to the bilayer-stitching $\mathrm{O}-\mathrm{H}$ stretch vibrations of water molecules in the top two bilayers of the 

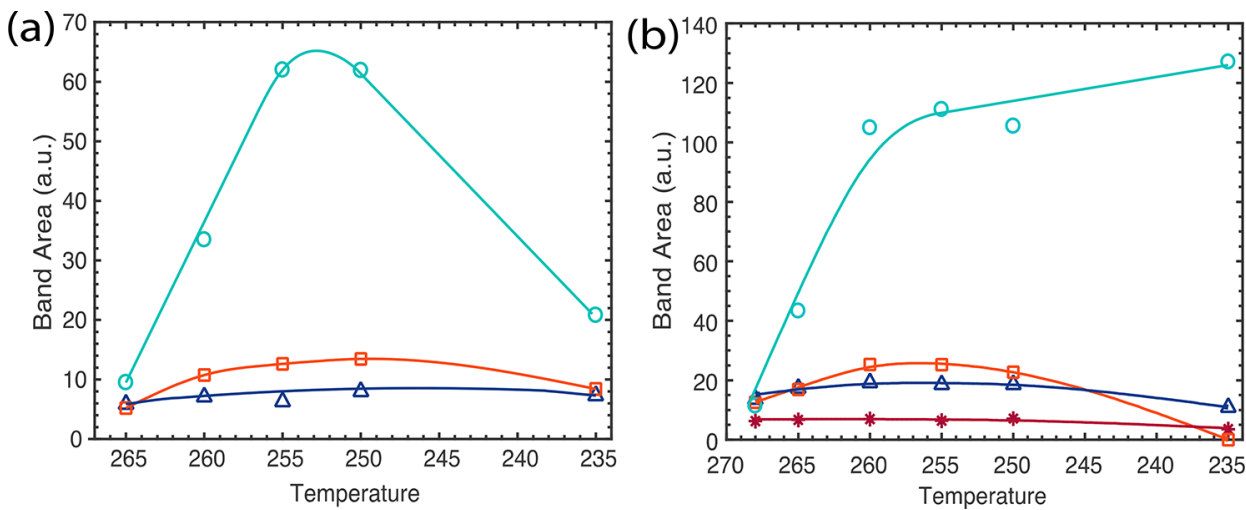

Figure 5. Areas of the different bands of the spectral decomposition of the temperature-dependent $\operatorname{Im}\left[\chi^{(2)}\right]$ spectra of (a) AAc and (b) PAc. The different bands are assigned to the crystalline ice signal at $\sim 3160 \mathrm{~cm}^{-1}$ (cyan $\mathrm{O}$ ), to strongly hydrogen-bonded OH groups of the carboxylic acid molecules at $\sim 3080 \mathrm{~cm}^{-1}$ (orange $\square$ ), to the $\mathrm{CH}_{2}$ of the PAc at $2880 \mathrm{~cm}^{-1}$ (red *), and to the $\mathrm{CH}_{3}$ vibrations of the methyl group of the carboxylic acids at $\sim 2950^{-1} \mathrm{~cm}($ dark blue $\triangle)$. The solid lines are a guide to the eye.

ice crystal. ${ }^{16,26}$ We observe that the peak of the bilayerstitching $\mathrm{O}-\mathrm{H}$ stretch vibrations of the ice is red-shifted in comparison to the bare ice surface. This indicates that the strength of the hydrogen bonds of the water molecules at the surface is increased, as has also been observed for other systems. ${ }^{18}$ For the solution of PAc on ice, we include an additional component representing the $\mathrm{C}-\mathrm{H}$ vibration of the $\mathrm{CH}_{2}$ group (band at $2880 \mathrm{~cm}^{-1}$ ).

It should be noted that the studied solutions will contain ions as a result of acid dissociation. In the studied systems, the concentrations of ions will be low, in view of the $\mathrm{p} K_{\mathrm{a}}$ values $\left(\mathrm{p} K_{\mathrm{a}}=4.76\right.$ for AAc and $\mathrm{p} K_{\mathrm{a}}=4.87$ for $\left.\mathrm{PAc}^{29,30}\right)$. For a $40 \mathrm{wt}$ $\%(=11.8 \mathrm{M})$ acetic acid $\left(\mathrm{CH}_{3} \mathrm{COOH}\right)$ solution, the concentrations $\left[\mathrm{H}_{3} \mathrm{O}^{+}\right]$and $\left[\mathrm{CH}_{3} \mathrm{COO}^{-}\right]$are $14 \mathrm{mM}$. In case the acetate/propionate ions would have a propensity to accumulate at the interface of the ice crystal and the carboxylic acid solution, this would create a static electric field at the surface that orients the water molecules in the ice close to the surface, which will lead to a field-induced $\chi^{(3)}$ contribution to the SFG signal. This surface field-induced $\chi^{(3)}$ contribution has been studied in detail both theoretically and experimentally. $^{26-28}$ In the studied systems, an electric field originating from the accumulation of acetate/propionate ions at the icesolution interface would be short-range as it is effectively screened by protons that diffuse into the ice crystal.

The screening by the protons diffused into the ice will be very effective as compared to Debye screening. In the case of Debye screening, the screening results from a difference in the distribution of the positive and negative ions in solution, with an associated energy penalty for their separation. In this case, this penalty is absent as only the positive ions will diffuse into the ice. For a solution of $14 \mathrm{mM}$ of positive and negative ions, the Debye length is $2.5 \mathrm{~nm}$. Hence, the length over which the protons screen the negative surface charge is expected to be shorter than $2.5 \mathrm{~nm}$. The coherence length in SFG in reflection geometry is typically several tens of nanometers, corresponding to $>100$ layers of water molecules. ${ }^{27}$ Hence, the depth over which water molecules may be oriented due to a surface electric field will be much smaller than the coherence length, which implies that the field-dependent $\chi^{(3)}$ contribution will be in phase with the $\chi^{(2)}$ contribution. This means that the VSFG signal of the ice-solution interface will not be distorted by this contribution.
This latter notion is confirmed by our finding that we can decompose the observed VSFG spectra very well into a contribution of the solution-air interface and that of an ice surface with a response that is similar in shape to that of a bare ice-air interface. This indicates that the response of the ice is not distorted as a result of a field-dependent $\chi^{(3)}$ contribution.

In Figure 5 we plot the areas of the bands as a function of temperature. We find that the band areas of the $\mathrm{CH}_{3}$ and $\mathrm{CH}_{2}$ vibrations are almost temperature independent. This result agrees with the results of Tyrode and co-workers for a solution of AAc at room temperature. ${ }^{25}$ In this latter study, the $\mathrm{CH}_{3}$ signal was observed to quickly saturate when the concentration of AAc was increased. This saturation indicates that already at relatively low bulk concentrations the surface gets completely covered with AAc molecules. According to the phase diagram shown in Figure 3c, a liquid aqueous solution of AAc at $265 \mathrm{~K}$ contains a concentration of $\sim 22$ wt $\%$ AAc, which can be considered as a high concentration in comparison with the results of Tyrode and co-workers. Hence, already at $265 \mathrm{~K}$ the surface will be saturated with AAc molecules, and decreasing the temperature toward the eutectic temperature will increase the concentration of AAc in the bulk of the AAC solution on top of the ice crystal, but not at the surface of the solution. The same effect occurs for the PAc solution on the ice surface; already at $268 \mathrm{~K}$ the surface of the PAc solution will be saturated with PAc molecules, and decreasing the temperature will not further increase the surface concentration. This means that for temperatures above the eutectic point, the $\mathrm{CH}$ response of an acid solution on top of ice is similar to that of an aqueous acid solution at room temperature. Cooling the systems below the eutectic point leads to a slight decrease in the band areas of the $\mathrm{C}-\mathrm{H}$ vibrations, which indicates a change in orientation of the AAc and PAc molecules when cooling below the eutectic point.

For both AAc and PAc, the band area of the $\mathrm{O}-\mathrm{H}$ stretch vibration of the carboxylic acid group increases with decreasing temperature in a temperature interval above the eutectic point. This observation indicates that the response of this vibration follows the bulk concentration of AAc/PAc in the solution on top of the ice surface that increases due to molecular freeze concentration. This result suggests that this response not only results from the (saturated) top molecular layer of the aqueous solution but also from layers underneath this layer and from layers close to the ice surface. When the temperature is 
lowered below the eutectic point, the amplitude of the $\mathrm{OH}$ vibration of the carboxylic acid group decreases somewhat for AAc, whereas for PAc this contribution completely vanishes, indicating a significant change of the interactions between the molecules for the latter system.

The band area of the crystalline ice signal at $3160 \mathrm{~cm}^{-1}$ strongly increases when the temperature is decreased, for solutions of both AAc and PAc on top of the ice crystal. This increase is also observed for the interface of bare ice and air and has been explained from the enhanced ordering of the water molecules in the top molecular layers of the ice crystal surface. $^{31}$

For the AAc system, the crystalline ice signal at $3160 \mathrm{~cm}^{-1}$ dominates the VSFG signal of the $\mathrm{O}-\mathrm{H}$ vibrations already at a temperature of $260 \mathrm{~K}$. As a result, the $\mathrm{O}-\mathrm{H}$ VSFG signal has a temperature dependence that closely resembles that of a crystalline ice-air interface, showing a small red-shift when the temperature is decreased. For the PAc system, the carboxylic acid group significantly contributes to the overall O-H VSFG signal, probably because the PAc solution on top of ice contains a higher mole fraction of carboxylic acid molecules than does a solution of AAc on top of ice at the same temperature (Figures $3 \mathrm{c}$ and $4 \mathrm{c}$ ). With decreasing temperature, the amplitude of the carboxylic acid component increases less strongly than the crystalline ice component. As a result, for the PAc system, the overall O-H VSFG signal undergoes a blueshift when the temperature is decreased toward the eutectic point.

There is a large difference between the AAc and PAc systems in how the $\mathrm{O}-\mathrm{H}$ VSFG signal changes when the temperature is lowered below the eutectic point. For AAc the signal decreases, while keeping the same shape. The surface above the eutectic point is smooth, and no scattering is observed. When we cool the AAc system below the eutectic point, we observe that the surface starts to show diffuse light scattering, due to roughening of the surface. Furthermore, we observe an overall decrease of the intensity in all frequencies of the VSFG spectrum, but no change of the shape of this spectrum. For PAc we observe a further increase of the amplitude and an additional blue-shift when the temperature is lowered below the eutectic point.

From the analysis of the $\operatorname{Im}\left[\chi^{(2)}\right]$ VSFG spectrum of the PAc solution on top of ice at $235 \mathrm{~K}$ (Figures $4 \mathrm{~b}$ and $5 \mathrm{~b}$ ), it follows that the carboxylic acid contribution to the O-H VSFG signal vanishes completely below the eutectic point. As a result, the frequency and shape of the observed signal are almost identical to those of a pure ice-air interface at $245 \mathrm{~K}$. We explain the intensity increase of the crystalline ice signal and vanishing of the carboxylic acid contribution from a phase separation of the water and propionic acid molecules upon freezing of the eutectic PAc-water mixture. ${ }^{32,33}$ The vanishing of the VSFG signal of the PAc molecules suggests that these molecules cluster in hydrogen-bonded dimers with an opposite alignment of the carboxylic acid $\mathrm{O}-\mathrm{H}$ groups. As a result, the $\mathrm{OH}$ groups of the strongly hydrogen-bonded carboxylic acids no longer show a net orientation, and the VSFG signal vanishes. This explanation is supported by previous work showing that PAc indeed forms cyclic dimers upon crystallization. ${ }^{34,3}$

\section{CONCLUSIONS}

We studied the properties of aqueous AAc and PAc acid solutions at the surface of ice at different temperatures using HD-VSFG spectroscopy. This technique allows us to determine $\left|\chi^{(2)}\right|^{2}, \operatorname{Im}\left[\chi^{(2)}\right]$, and $\operatorname{Re}\left[\chi^{(2)}\right]$. The VSFG spectra show resonances that can be assigned to the $\mathrm{C}-\mathrm{H}$ stretch vibration of the methyl group of acetic/propionic acid (2950 $\mathrm{cm}^{-1}$ ), the $\mathrm{C}-\mathrm{H}$ stretch vibration of the methylene group of PAc $\left(2880 \mathrm{~cm}^{-1}\right)$, the $\mathrm{O}-\mathrm{H}$ vibration of the carboxylic acid group $\left(3080 \mathrm{~cm}^{-1}\right)$, and the $\mathrm{O}-\mathrm{H}$ vibrations $\left(3160 \mathrm{~cm}^{-1}\right)$ of the ice surface underneath the aqueous acetic/propionic acid solution.

Upon deposition of concentrated acid solutions on the basal surface of ice, the ice melts until a concentration is reached that is stable according to the phase diagram of the mixture. Lowering the temperature causes a freeze-induced phase separation (FIPS) into pure ice and a freeze concentrated acid solution (FCS) for both AAc and PAc.

For the AAc solution, lowering the temperature below the eutectic point leads to a decrease of the VSFG signal while retaining the shape of the spectrum, indicating a roughening of the surface of the frozen solution and enhanced diffuse scattering. Cooling of the PAc solution on top of ice below the eutectic point leads to a strong VSFG signal that is highly similar to that of a pure ice-air interface at the same temperature. This observation indicates the formation of hydrogen-bonded dimers of propionic acid molecules with an opposite alignment of their carboxylic acid $\mathrm{O}-\mathrm{H}$ groups, leading to a vanishing of the carboxylic acid contribution to the $\mathrm{O}-\mathrm{H}$ VSFG signal.

\section{ASSOCIATED CONTENT}

\section{(s) Supporting Information}

The Supporting Information is available free of charge at https://pubs.acs.org/doi/10.1021/acs.jpcb.9b10462.

Figure S1, Modeling of propionic acid at the surface of ice with different solution layer thicknesses; Figure S2, Maximized imaginary spectrum of propionic acid at the surface of ice at $268 \mathrm{~K}$; Figure S3, $\operatorname{Re}\left[\chi^{(2)}\right]$ of aqueous acetic acid (40 wt \%, red) at the surface of ice at different temperatures; Figure S4, Reversibility of the VSFG signals upon cooling below the eutectic point of AAc; Figure $S 5, \operatorname{Re}\left[\chi^{(2)}\right]$ of aqueous propionic acid $(40$ wt $\%$, green) at the surface of ice at different temperatures; Figure S6, Reversibility of the VSFG signals upon cooling below the eutectic point of PAc; and Figure S7, HD-VSFG spectrum of monocrystalline basal plane ice (PDF)

\section{AUTHOR INFORMATION}

\section{Corresponding Author}

Carolyn J. Moll - AMOLF, Amsterdam 1098 XG, The

Netherlands; (1) orcid.org/0000-0001-6041-5898;

Email: cmoll@amolf.nl

\section{Authors}

Konrad Meister - AMOLF, Amsterdam 1098 XG, The Netherlands; Max-Planck Institute for Polymer Research, Mainz D 55128, Germany; University of Alaska Southeast, Juneau, Alaska 99801, United States; 이이.org/0000-0002-68536325

Jan Versluis - AMOLF, Amsterdam 1098 XG, The Netherlands Huib J. Bakker - AMOLF, Amsterdam 1098 XG, The

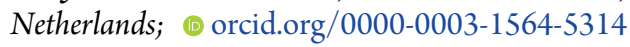

Complete contact information is available at: https://pubs.acs.org/10.1021/acs.jpcb.9b10462 


\section{Funding}

This project has received funding from the European Research Council (ERC) under the European Union's Horizon 2020 research and innovation program (grant agreement No 694386).

\section{Notes}

The authors declare no competing financial interest.

\section{ACKNOWLEDGMENTS}

This work is part of the research program of The Netherlands Organization for Scientific Research (NWO) and was performed at the research institute AMOLF.

\section{REFERENCES}

(1) Chebbi, A.; Calier, P. Carboxylic Acids in the Troposphere, Occurrence, Sources, and Sinks: A Review. Atmos. Environ. 1996, 30 (24), 4233-4249.

(2) Talbot, R. W.; Dibb, J. E.; Lefer, B. L.; Scheuer, E. M.; Bradshaw, J. D.; Sandholm, S. T.; Smyth, S.; Blake, D. R.; Blake, N. J.; Sachse, G. W.; Collins, J. E.; Gregory, G. L. Large-Scale Distributions of Tropospheric Nitric, Formic, and Acetic Acids over the Western Pacific Basin during Wintertime. J. Geophys. Resaerch 1997, 102 (28), 303-28.

(3) Bogdan, A. Ice Clouds: Atmospheric Ice Nucleation Concept versus the Physical Chemistry of Freezing Atmospheric Drops. J. Phys. Chem. A 2018, 122 (39), 7777-7781.

(4) Bogdan, A.; Molina, M. J.; Tenhu, H.; Mayer, E.; Loerting, T. Formation of Mixed-Phase Particles during the Freezing of Polar Stratospheric Ice Clouds. Nat. Chem. 2010, 2 (3), 197-201.

(5) Bogdan, A.; Molina, M. J. Physical Chemistry of the Freezing Process of Atmospheric Aqueous Drops. J. Phys. Chem. A 2017, 121 (16), 3109-3116.

(6) Bogdan, A.; Molina, M. J. Aqueous Aerosol May Build up an Elevated Upper Tropospheric Ice Supersaturation and Form MixedPhase Particles after Freezing. J. Phys. Chem. A 2010, 114 (8), 28212829.

(7) Cox, R. A.; Fernandez, M. A.; Symington, A.; Ullerstam, A.; Abbatt, J. P. D. A kinetic model for uptake of $\mathrm{HNO}_{3}$ and $\mathrm{HCl}$ on ice in a coated wall flow system. Phys. Chem. Chem. Phys. 2005, 7, 34343442 .

(8) Grannas, A. M.; Bogdal, C.; Hageman, K. J.; Halsall, C.; Harner, T.; Hung, H.; Kallenborn, R.; Klan, P.; Klanova, J.; Macdonald, R. W.; Meyer, T.; Wania, F. The Role of the Global Cryosphere in the Fate of Organic Contaminants. Atmos. Chem. Phys. 2013, 13, 3271-3305.

(9) von Hessberg, P.; Pouvesle, N.; Winkler, A. K.; Schuster, G.; Crowley, J. N. Interaction of Formic and Acetic Acid with Ice Surfaces between 187 and $227 \mathrm{~K}$. Investigation of Single Species- and Competitive Adsorption. Phys. Chem. Chem. Phys. 2008, 10, 23452355.

(10) Steffen, A.; Douglas, T.; Amyot, M.; Ariya, P.; Aspmo, K.; Berg, T.; Bottenheim, J.; Brooks, S.; Cobbett, F.; Dastoor, A.; Dommergue, A.; Ebinghaus, R.; Ferrari, C.; Gardfeldt, K.; Goodsite, M. E.; Lean, D.; Poulain, A. J.; Scherz, C.; Skov, H.; Sommar, J.; Temme, C. A Synthesis of Atmospheric Mercury Depletion Event Chemistry in the Atmosphere and Snow. Atmos. Chem. Phys. 2008, 8, 1445-1482.

(11) Bogdan, A.; Molina, M. J.; Kulmala, M.; Tenhu, H.; Loerting, T. Solution Coating around Ice Particles of Incipient Cirrus Clouds. Proc. Natl. Acad. Sci. U. S. A. 2013, 110 (27), 2439.

(12) Waldner, A.; Artiglia, L.; Kong, X.; Orlando, F.; Huthwelker, T.; Ammann, M.; Bartels-Rausch, T. Pre-Melting and the Adsorption of Formic Acid at the Air - Ice Interface at $253 \mathrm{~K}$ as Seen by NEXAFS and XPS. Phys. Chem. Chem. Phys. 2013, 00, 1-3.

(13) Anim-Danso, E.; Zhang, Y.; Dhinojwala, A. Freezing and Melting of Salt Hydrates Next to Solid Surfaces Probed by Infrared Visible Sum Frequency Generation Spectroscopy. J. Am. Chem. Soc. 2013, 135, 8496.
(14) Shen, Y. R. Surface Properties Probed Ny Second-Harmonic and Sum-Frequency Generation. Nature 1989, 337, 519-525.

(15) Anim-Danso, E.; Zhang, Y.; Alizadeh, A.; Dhinojwala, A. Freezing of Water Next to Solid Surfaces Probed by Infrared - Visible Sum Frequency Generation Spectroscopy. J. Am. Chem. Soc. 2013, 135, 2734-2740.

(16) Smit, W. J.; Tang, F.; Nagata, Y.; Sánchez, M. A.; Hasegawa, T.; Backus, E. H. G.; Bonn, M.; Bakker, H. J. Observation and Identification of a New OH Stretch Vibrational Band at the Surface of Ice. J. Phys. Chem. Lett. 2017, 8 (15), 3656-3660.

(17) Wei, X.; Miranda, P. B.; Shen, Y. R. Surface Vibrational Spectroscopic Study of Surface Melting of Ice. Phys. Rev. Lett. 2001, 86 (8), 1554-1557.

(18) Meister, K.; Moll, C. J.; Chakraborty, S.; Jana, B.; DeVries, A. L.; Ramløv, H.; Bakker, H. J. Molecular Structure of a Hyperactive Antifreeze Protein Adsorbed to Ice. J. Chem. Phys. 2019, 150 (13), 131101.

(19) Cyran, J. D.; Backus, E. H. G.; van Zadel, M. J.; Bonn, M. Comparative Adsorption of Acetone on Water and Ice Surfaces. Angew. Chem., Int. Ed. 2019, 58 (11), 3620-3624.

(20) Shen, Y. R. Phase-Sensitive Sum-Frequency Spectroscopy. Annu. Rev. Phys. Chem. 2013, 64, 129.

(21) Roos, D. v. D. Rapid Production of Single Crystals of Ice. J. Glaciol. 1975, 14 (71), 325-328.

(22) Fairbrain, H. W. Structural Petrology of Deformed Rocks; Addison-Wesley: Boston, MA, 1954.

(23) Hill, I. R.; Levin, I. W. Vibrational Spectra and CarbonHydrogen Stretching Mode Assignments for a Series of n -Alkyl Carboxylic Acids. J. Chem. Phys. 2003, 842 (1979), 842.

(24) Johnson, C. M.; Tyrode, E.; Baldelli, S.; Rutland, M. W. A Vibrational Sum Frequency Spectroscopy Study of the Liquid - Gas Interface of Acetic Acid - Water Mixtures: 1. Surface Speciation. J. Phys. Chem. B 2005, 109, 321-328.

(25) Tyrode, E.; Johnson, C. M.; Baldelli, S.; Rutland, M. W. A Vibrational Sum Frequency Spectroscopy Study of the Liquid-Gas Interface of Acetic Acid-Water Mixtures: 2. Orientation Analysis. J. Phys. Chem. B 2005, 109, 329-341.

(26) Yuji, O.; Sugimoto, T.; Ishiyama, T.; Morita, A.; Watanabe, K.; Matsumoto, Y. Unveiling Subsurface Hydrogen-Bond Structure of Hexagonal Water Ice. Phys. Rev. B: Condens. Matter Mater. Phys. 2017, $115405,1-14$.

(27) Reddy, S. K.; Thiraux, R.; Wellen Rudd, B. A.; Lin, L.; Adel, T.; Joutsuka, T.; Geiger, F. M.; Allen, H. C.; Morita, A.; Paesani, F. Bulk Contributions Modulate the Sum-Frequency Generation Spectra of Water on Model Sea-Spray Aerosols. Chem. 2018, 4 (7), 1629-1644.

(28) Ohno, P. E.; Wang, H. F.; Geiger, F. M. Second-Order Spectral Lineshapes from Charged Interfaces. Nat. Commun. 2017, 8 (1), 1-9. (29) Ohno, P. E.; Wang, H. F.; Paesani, F.; Skinner, J. L.; Geiger, F. M. Second-Order Vibrational Lineshapes from the Air/Water Interface. J. Phys. Chem. A 2018, 122 (18), 4457-4464.

(30) Saracino, G. A. A.; Improta, R.; Barone, V. Absolute PKa Determination for Carboxylic Acids Using Density Functional Theory and the Polarizable Continuum Model. Chem. Phys. Lett. 2003, 373 (3-4), 411-415.

(31) Smit, W. J.; Bakker, H. J. The Surface of Ice Is Like Supercooled Liquid Water. Angew. Chem., Int. Ed. 2017, 56 (49), $15540-15544$.

(32) Bogdan, A.; Molina, M. J.; Tenhu, H.; Mayer, E.; Bertel, E.; Loerting, T. Different Freezing Behavior of Millimeter-and Micrometer-Scaled (NH 4)2SO4/H2O Droplets. J. Phys.: Condens. Matter 2011, 23 (3), 035103.

(33) Bogdan, A. Double Freezing of (NH4)2SO4/H $2 \mathrm{O}$ Droplets below the Eutectic Point and the Crystallization of (NH4)2SO4 to the Ferroelectric Phase. J. Phys. Chem. A 2010, 114 (37), 1013510139.

(34) Cyriac, J.; Pradeep, T. J. Phys. Chem. C 2008, 112, 1604-1611. (35) Sander, W.; Gantenberg, M. Aggregation of Acetic and Propionic Acid in Argon Matrices - A Matrix Isolation and 
Computational Study. Spectrochim. Acta, Part A 2005, 62 (4-5), 902-909.

(36) Barr, R. S.; Newsham, D. M. T. Freezing Temperatures of Water, Alkanoic Acids and Their Mixtures. Chem. Eng. J. 1986, 33, 79-86. 